\title{
Residual stress evaluation in composites using a modified layer removal method
}

\author{
M. P. I. M. Eijpe \& P. C. Powell \\ Department of Mechanical Engineering, University of Twente (UT), Enschede, The Netherlands
}

\begin{abstract}
The layer removal method is often used for measurement of internal stresses in homogeneous polymeric materials. In order to extend the use of this method to laminated composites certain refinements are needed. These include: (i) use of varying material properties (elastic moduli) through the thickness of the composite plate; (ii) use of geometric non-linear analysis to account for large deformations; and (iii) measurement not only of curvatures but also of strains. These refinements are necessary because a non-symmetric laminate is created when layers are removed, which shows large curvatures.

The modified layer removal method was theoretically validated on a typical compression-moulded continuous-fibre laminate (PEI/glass) and a iypical injection-moulded short-fibre-reinfonced laminate (PC/glass). The modified method produced good results and the need to use the modified layer removal analysis is clearly demonstrated. (C) 1997 Elsevier Science Ltd.
\end{abstract}

\section{INTRODUCTION}

Plastics reinforced with continuous and short fibres, viz. composite materials, are used increasingly in non-structural as well as structural applications. Almost every composite product produced above room temperature, and used at room temperature, contains residual stresses. The major cause for these residual stresses is the difference in the thermal expansion coefficient between fibre and matrix in combination with a high processing temperature and a varying fibre orientation through the thickness of a product. These stresses are referred to as thermal anisotropy stresses. A knowledge of the residual stresses is important for reliable strength assessment. They use up a part of the inherent material strength. Many cases are reported in literature where the thermal anisotropy stresses use up more than half the matrix strength $[1,2]$.

Composite designers have developed methods for simulating residual stresses. Often the 'classical lamination theory' (CLT) is used. However, there is a lack of experimental validation proving the prediction to be correct. For experimental validation, a reliable technique is needed.

An established method for experimental evaluation of residual stresses in homogeneous isotropic materials is the layer removal method [3]. However, the layer removal method in its present form cannot be used on composite materials with a variation in properties through its thickness. Paterson \& White $[4,5]$ developed the layer removal method for use on plates with a depth-varying modulus. But, in their final analysis, they do not consider the fact that in composite products the Poisson's ratios can also vary considerably through the thickness.

In addition, in composite plates, the curvatures after layer removal can become large. Where this occurs, the CLT no longer provides the correct basis for calculations. Hyer $[6,7]$, Jun \& Hong [8,9] and Peeters et al. [10] show that large (thermal) curvatures in non-symmetric composite plates cannot be calculated using the CLT but that an energy approach can be used.

In this research, a modified layer removal method was developed in which variation of mechanical properties through the thickness is 
taken into account and in which an energy approach is used to account for the large curvatures resulting from successive layer removal. The derivations are performed for crossply laminates.

The modified layer removal method is validated for a polyetherimide (PEI) continuousglassfibre-reinforced laminate and a polycarbonate (PC) short-glassfibre-reinforced laminate.

\section{LAYER REMOVAL METHOD}

In the layer removal method, thin layers are machined from one surface of a plate and the curvature that is produced to restore force equilibrium is measured at each incremental removal. The curvature profile against thickness removed can then be used to derive the stress profile through the thickness in the original plate. The layer removal analysis can be divided into two parts. The first part deals with the relation between the deformations after layer removal and the induced force and moment resultants. This part is material and sample-geometry dependent. The second part is the relation between the induced moment resultants $\left(M_{i}\right)$ and the stresses $\left(\sigma_{i}\right)$ in the original sample. This part is independent of material or sample geometry and was derived by Treuting $\&$ Read [3]. For the $x$-direction, it is given by

$$
\begin{aligned}
\sigma_{x}\left(z_{1}\right) & =\frac{2}{z_{0}+z_{1}} \frac{\mathrm{d} M_{x}\left(z_{1}\right)}{\mathrm{d} z_{1}}+\frac{2 M_{x}\left(z_{1}\right)}{\left(z_{0}+z_{1}\right)^{2}} \\
& -4 \int_{z_{1}}^{z_{1}} \frac{M_{x}(z)}{\left(z_{0}+z^{3}\right)^{3}} \mathrm{~d} z
\end{aligned}
$$

The co-ordinates definition used is given in Fig. 1. The relation between the induced moment resultants and the deformations after layer removal for composite plates will be derived in the next section.

\section{THEORETICAL ANALYSIS}

The CLT [11] provides a relation between moment resultants per unit width $\left(M_{i}\right)$ and midplane strains $\left(\varepsilon_{\mathrm{i}}^{0}\right)$ and curvatures $\left(\kappa_{\mathrm{i}}\right)$ for a crossply laminate. The moment resultant per unit width in the $x$-direction is given by

$$
M_{x}=B_{11} \kappa_{x}^{0}+D_{11} \kappa_{x}+D_{12} \kappa_{y}
$$
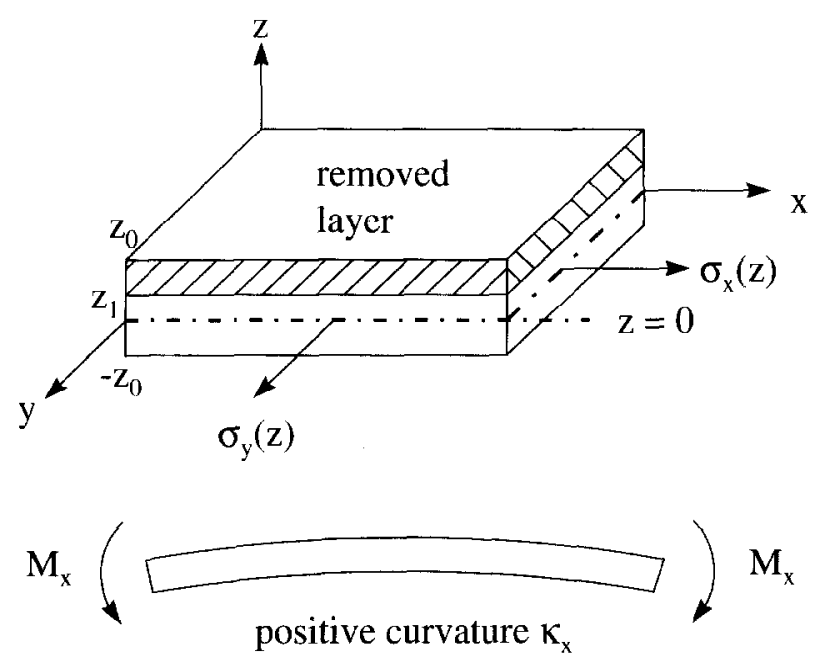

Fig. 1. Co-ordinate system used in layer removal analysis.

where $B_{11}, D_{11}$ and $D_{12}$ are stiffness coefficients as defined in the CLT. Using eqn (2), the variation in mechanical properties through the thickness is accounted for.

The CLT is strictly valid for small deformations only.

A different approach is needed if the curvatures after layer removal become large. Non-linear terms in the strain-displacement relations can no longer be neglected, as is done in the CLT. Accounting for these terms is done using an energy approach described by Jun \& Hong [8,9] and Peeters et al. [10]. The Rayleigh-Ritz method [12] is used to obtain solutions. In this approach, the stored potential energy in the system is calculated as a function of unknown Ritz coefficients $\left(r_{i}\right)$ from an assumed displacement field. This system is in a stable equilibrium when the potential energy is at a minimum.

This minimum can be found by minimizing the potential energy $(U)$ according to the Ritz coefficients $r_{i}$ :

$$
\mathrm{d} U=\sum_{i}\left(\frac{\partial U}{\partial r_{i}}\right) \partial r_{i}=0
$$

This 'minimum potential energy' (MPE) approach will be referred hereafter as MPE. The potential energy of a plate from which part is machined off is given (in contracted notation) by

$$
U=\int_{V}\left(\frac{1}{2} Q_{i j}^{*} \varepsilon_{i} \varepsilon_{j}-\sigma_{j} \varepsilon_{j}\right) \mathrm{d} V \text { with } i, j=x, y, x y
$$


in which $\sigma_{j}$ are the stresses in the sample before layer removal, $\varepsilon_{i}$ and $\varepsilon_{j}$ are the strains in the sample after layer removal and $Q_{i j}^{*}$ are the transformed stiffness coefficients for a unidirectional ply as defined in the CLT. Carrying out the integration over the thickness in eqn (4) and using $\varepsilon_{\mathrm{i}}(\mathrm{z})=\varepsilon_{\mathrm{i}}^{0}+\mathrm{z} \kappa_{\mathrm{i}}$ gives

$$
\begin{aligned}
U= & \iint\left(\frac{1}{2} A_{i j} \varepsilon_{i}^{0} \varepsilon_{j}^{0}+B_{i j} \varepsilon_{i}^{0} \kappa_{j}+\frac{1}{2} D_{i j} \kappa_{i} \kappa_{j}-N_{j} \varepsilon_{j}^{0}\right. \\
& \left.-M_{j} \kappa_{j}\right) \mathrm{d} x \mathrm{~d} y \text { with } i, j=x, y, x y
\end{aligned}
$$

in which $A_{i j}, B_{i j}$ and $D_{i j}$ are the laminate stiffness coefficients as defined in the CLT, and $N_{j}$ and $M_{j}$ are, respectively, the force and moment resultants per unit width induced by layer removal. For a crossply laminate containing only thermal direct stresses, $N_{i}$ and $M_{i}$ will be $N_{x}, N_{y}, M_{x}$ and $M_{y}$. In the CLT, the indices $i$ or $j$ in the stiffnesses $A_{i j}, B_{i j}$ and $D_{i j}$ can be 1,2 or 6. This means that, for example, $A_{x x}$ defined in eqn (5) is $A_{11}$ in the CLT and $A_{x x y}$ is $A_{16}$. Note that this is not true for $\sigma_{i}, \varepsilon_{i}, N_{i}$ or $M_{i}$. For example $\sigma_{i} \neq \sigma_{x}$.

A modified von Karman approximation to Greene's strains [8] gives the following straindisplacement relations, in which the right-hand side terms between brackets are the non-linear terms neglected in the CLT

$$
\begin{aligned}
& \varepsilon_{x}(z)=\frac{\mathrm{d} u}{\mathrm{~d} x}+\frac{1}{2}\left(\frac{\mathrm{d} w}{\mathrm{~d} x}\right)^{2}+z \frac{\mathrm{d}^{2} w}{\mathrm{~d} x^{2}} \\
& \varepsilon_{y}(z)=\frac{\mathrm{d} v}{\mathrm{~d} y}+\frac{1}{2}\left(\frac{\mathrm{d} w}{\mathrm{~d} y}\right)^{2}+z \frac{\mathrm{d}^{2} w}{\mathrm{~d} y^{2}} \\
& \varepsilon_{x y}(z)=\frac{\mathrm{d} u}{\mathrm{~d} y}+\frac{\mathrm{d} v}{\mathrm{~d} x}+\left(\frac{\mathrm{d} w}{\mathrm{~d} x} \frac{\mathrm{d} w}{\mathrm{~d} y}\right)+2 z \frac{\mathrm{d}^{2} w}{\mathrm{~d} x \mathrm{~d} y}
\end{aligned}
$$

Here $u, v$ and $w$ are the displacements in the $x$-, $y$ - and $z$-directions, $\varepsilon_{x}$ and $\varepsilon_{y}$ the strains in the $x$ and $y$-directions, and $\varepsilon_{y y}$ is twice the shear strain as normally defined. This means that $\varepsilon_{x y}$ is $\gamma_{x y}$ which is always used in the CLT.

The displacement field as given by Jun \& Hong [8,9] and Peeters et al. [10] is

$$
\begin{aligned}
& u(x, y)=x\left(a_{1}-\frac{a^{2}}{6} x^{2}+a_{3} y^{2}\right) \\
& v(x, y)=y\left(b_{1}-\frac{b^{2}}{6} y^{2}+b_{3} x^{2}\right) \\
& w(x, y)=\frac{1}{2}\left(a x^{2}+b y^{2}\right)
\end{aligned}
$$

where $a, a_{1}, a_{3}, b, b_{1}$ and $b_{3}$ are the unknown Ritz coefficients earlier indicated as $r_{i}$. Here $a$ and $b$ are, respectively, the curvatures $\kappa_{x}$ and $\kappa_{y}$, and $a_{1}$ and $b_{1}$ are the midplane strains $\varepsilon_{\mathrm{x}}^{0}$ and $\varepsilon_{\mathrm{y}}^{0}$ at $x=y=0$. Substitution of eqns (6) and (7) in eqn (5) gives an expression for the potential energy in terms of the unknown Ritz coefficients. To minimize the potential energy with respect to the $r_{i}$, a set of six equations can be formulated as

$$
\frac{\partial U}{\partial r_{1}}\left(r_{i}\right)=0 \text { with } r_{i}=a, a_{1}, a_{3}, b, b_{1}, b_{3}
$$

Equation (8) contains 10 unknowns, viz. $a, a_{1}$, $a_{3}, b, b_{1}$ and $b_{3}$ and the force and moment resultants per unit width $N_{x}, N_{y}, M_{x}$ and $M_{y}$. To solve eqn (8) at least four unknown parameters will have to be determined. In addition to the curvatures in the $x$-and $y$-directions, the strains at the surface can also be measured and transformed into the midplane strains. Subsequently eqn (8) can be solved to yield $M_{x}$ and $M_{y}$. Computing this for every layer removed results in a moment resultant per unit width profile against thickness removed. This result can be used in eqn (1) to calculate the stress profile in the original sample.

\section{DETERMINATION OF STRESSES, STRAINS AND CURVATURES}

The major contribution to residual stresses in composites are the thermal anisotropy stresses as indicated in the Introduction. For validation purposes, they are now assumed to be the only stresses present. These residual stresses are calculated for validation samples as a reference using the MPE approach.

Also the curvature and strain profiles, that would have been obtained by layer removal experiments on these validation samples, are determined from which the stresses are computed using the modified layer removal analysis, taking account for large deformations and varying material properties through the thickness.

\section{Thermal anisotropy stresses}

For a symmetric laminate, the thermal anisotropy stresses can be calculated using the CLT. However, for non-symmetric laminates, where out-of-plane deformations can be large, the 
earlier described energy approach must be used. This energy approach gives the same results as the CLT for small or no out-of-plane deflections and therefore will also be used for calculations on symmetric samples.

Equation (5) provides the basis for the calculations using the energy approach of thermal curvatures and strains in laminates, which result from cooling the laminates from the processing temperature to room temperature. During cooling, stresses and deformations will start to develop from the stress-free temperature, which is often the glass temperature for amorphous thermoplastic resins and is generally lower than the processing (melt) temperature.

For calculation of the thermal strains and curvatures only $N_{i}$ and $M_{i}$ in eqn (5), induced by layer removal, have to be replaced by thermally induced force and moment resultants $N_{i}^{T}$ and $M_{i}^{T} . N_{i}^{T}$ and $M_{i}^{T}$ are given by

$$
\begin{aligned}
& N_{i}^{T}=\sum_{f=1}^{n}\left(Q_{i, j, f}^{*}\left(h_{f}-h_{f-1}\right) \int \alpha_{j, f} \mathrm{~d} T\right) \text { and } \\
& M_{i}^{T}=\sum_{f=1}^{n}\left(Q_{i j, f}^{*}\left(h_{f}^{2}-h_{f-1}^{2}\right) \int \alpha_{j, f} \mathrm{~d} T\right)
\end{aligned}
$$

where $n$ is the number of plies in the laminate, $h_{f}$ is the thickness co-ordinate of the top of ply $f$ and $\alpha_{i, f}$ are the thermal expansion coefficients of ply $f$ in the $x$-, $y$ - and $x y$-components. The thermal anisotropy stresses in each ply $f$ can subsequently be obtained from the unconstrained thermal strains $\left(\varepsilon_{i, f}^{T}=\int \alpha_{i, f} \mathrm{~d} T\right)$ for each individual ply $f$ and the thermal strains of the complete laminate $\left(\varepsilon_{i, L}^{T}(z)=\varepsilon_{i, L}^{0}+z \kappa_{i, L}^{T}\right)$ according to

$$
\sigma(z)_{t, f}^{T}=Q_{i j, f}^{*} \cdot\left(\varepsilon_{i, L}^{T}(z)-\varepsilon_{i, f}^{T}\right)
$$

\section{Curvature and strain profiles resulting from layer removal}

Layer removal on an initially symmetric laminate containing thermal anisotropy stresses means that a non-symmetric laminate is created that develops curvature and strain. These curvatures (Fig. 2, case a) are equivalent to the curvatures obtained by directly cooling such a non-symmetric laminate from stress-free temperature to room temperature (Fig. 2, case b). However, the strains are not the same. The strains in case (b) are equal to the strains in case (a) plus the initial strains in the original (a)
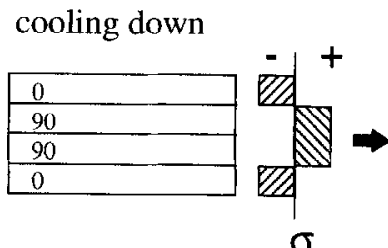

layer removal

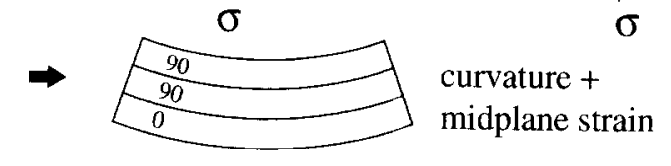

(b)

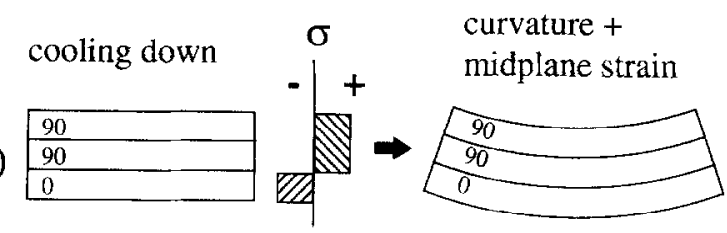

Fig. 2. Comparison between deformations caused by layer removal (top) and in directly cooling a non-symmetric laminate (bottom).

symmetric sample from which no layer is yet removed. This initial strain should therefore be subtracted from the strains determined in case (b).

\section{MATERIALS AND VALIDATION EXAMPLES}

Theoretical validation experiments were performed on laminates from two different materials. The first material is a polyetherimide (PEI) resin reinforced with $50 \%$ continuous glassfibre. This material is typically used in compression-moulding of plates, where crossply lay-ups are often used. The second material is a polycarbonate (PC) resin reinforced with $40 \%$ short glassfibre. This material is used in injection moulding. Injection-moulded plates can show skin core orientation with fibres aligned in the flow direction near the surface and aligned transverse to the flow direction in the core [13-16], which again is a typical crossply lay-up. The alignment of the fibres is caused by the mould geometry, the flow in the mould and the type of gating in the mould.

The required physical and mechanical properties of unidirectional plies made of the two materials are given in Table 1 .

The unidirectional plies of the two materials were used in four different laminates. These laminates were used in the validation to examine the influence of the material, the sample size and the laminate lay-up on the stresses in the $x$ - and $y$-directions calculated in the layer removal analysis. 
Table 1. Material properties of unidirectional plies used in the validation

\begin{tabular}{|c|c|c|c|c|c|c|c|}
\hline Material & $\begin{array}{c}E_{1} \\
(\mathrm{GPa})\end{array}$ & $\begin{array}{c}E_{2} \\
(\mathrm{GPa})\end{array}$ & $1 ' 12$ & $\begin{array}{c}G_{12} \\
(\mathrm{GPa})\end{array}$ & $\left(10^{\frac{\alpha_{1}}{6} / 0} \mathrm{C}\right)$ & $\left(10^{\alpha_{2} / \rho} \mathrm{C}\right)$ & $\begin{array}{c}T_{\mathrm{g}} \\
\left({ }^{\circ} \mathrm{C}\right)\end{array}$ \\
\hline $\mathrm{PEI} / 50 \%$ continuous glass & 43 & 14.3 & 0.27 & 5.5 & 8 & 22 & 215 \\
\hline PC/40\% short glass & 11.5 & 5 & 0.3 & 4 & 20 & 50 & 150 \\
\hline
\end{tabular}

(1) $(0,90)_{\text {sym }} \mathrm{PEI} /$ glass, dimensions $90 \times 30 \times$ $2 \mathrm{~mm}$, layer thicknesses $(0.5,0.5)_{\mathrm{sym}}$, ID code: pg93;

(2) $(0,90)_{\text {sym }} \mathrm{PEI} /$ glass, dimensions $100 \times 100 \times$ $2 \mathrm{~mm}$, layer thicknesses $(0.5,0.5)_{\mathrm{sym}}$, ID code: pg1010;

(3) $(0,90)_{\text {sym }}$ PC/glass, dimensions $90 \times 30 \times$ $2 \mathrm{~mm}$, layer thicknesses $(0.5,0.5)_{\mathrm{sym}}, \mathrm{ID}$ code: $\operatorname{cg} 93-55$;

(4) $(0,90)_{\text {sym }}$ PC/glass, dimensions $90 \times 30 \times$ $2 \mathrm{~mm}$, layer thicknesses $(0.7,0.3)_{\mathrm{sym}}$, ID code: $\operatorname{cg} 93-73$.

In addition, the influence of neglecting any of the input parameters in the analysis is examined. These input parameters are the curvatures and strains in the $x$ - and $y$-directions and the varying material properties through the thickness. Of the input parameters, either $\varepsilon_{x}$ or $\kappa_{y}$ is set to zero. Also, the material properties are assumed to be constant over the thickness. A typical $E$-modulus value of $29 \mathrm{GPa}$ and Poisson's ratio of 0.3 were used for analysis on sample pg93.

\section{RESULTS}

\section{Stresses for laminates 1-4}

The thermal anisotropy reference stresses in the chosen four laminates were calculated according to the MPE approach using eqn (10). The results are given in Fig. 3. The results are the same for the two PEI/glass laminates. The only difference between them is the size of the

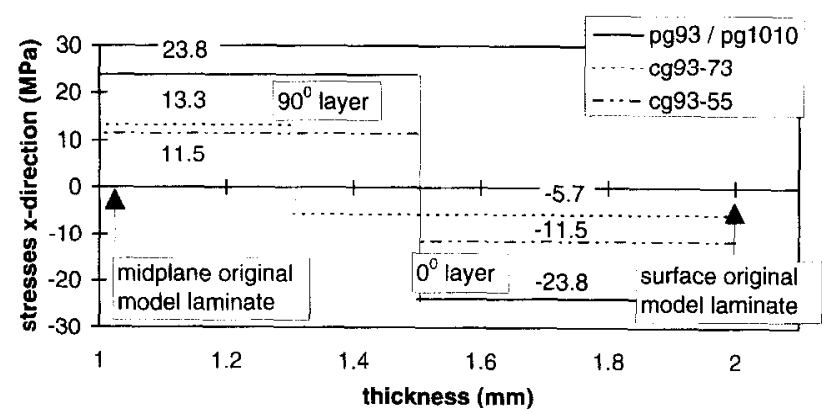

Fig. 3. Thermal anisotropy reference stresses in laminates $1-4$. samples, which has an influence only when curvature develops. Only half the laminate thickness is shown as the stress profile is symmetric with respect to the midplane.

\section{Curvature and strain profiles after layer removal}

The curvature and strain profiles resulting from layer removal experiments can be predicted theoretically by the MPE approach as the thermal curvatures and strains. These are calculated for the laminates that would have been obtained by successive layer removal of thin layers from the originally symmetric laminates. Layer removal was carried out for up to half the laminate thickness. Further layer removal is not performed as in these examples the stress profile is assumed to be symmetric with respect to the midplane of the original laminate.

The thermal midplane strains in the original symmetric laminate (curvatures are zero) are subtracted from the thermal strains in laminates from which layers are removed for reasons explained earlier.

The calculated thermal curvature profiles are shown in Fig. 4.

The calculated thermal midplane strain profiles are shown in Fig. 5.

\section{Moments}

From the curvature and strain profiles, the moment profiles as function of remaining lami-

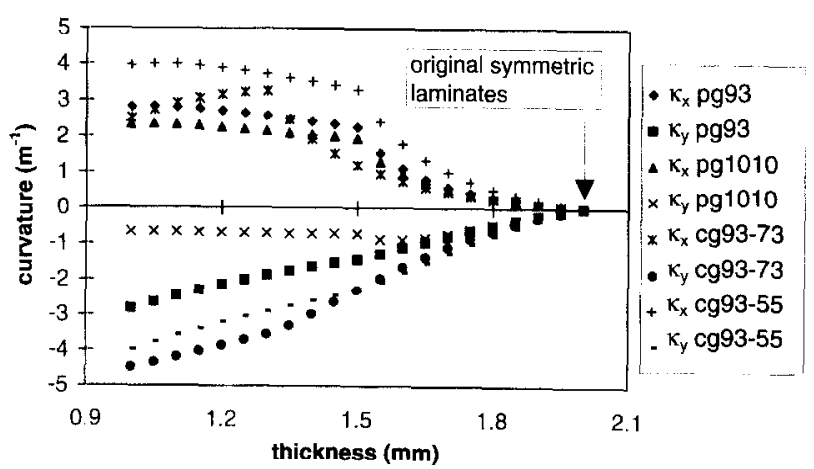

Fig. 4. Thermal curvature profiles (simulating layer removal). 


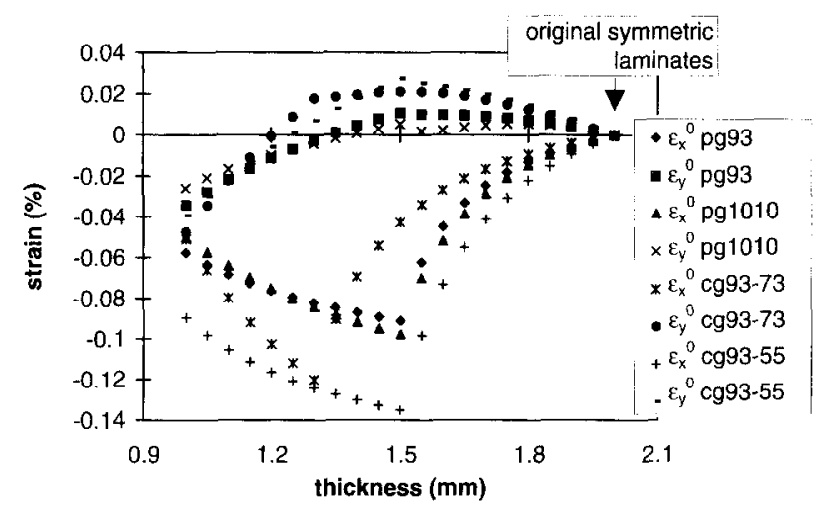

Fig. 5. Thermal midplane strain profiles (simulating layer removal).

nate thickness can be calculated. This was done using both the CLT (eqn (2)) and the MPE (eqn (5)). Results are shown in Figs 6-9. Leastsquares fitting was performed on the moment values, which was used to calculate the stresses. Within a layer of constant fibre orientation, a straight line is fitted. This will result in a constant stress within the layer. The fitting is not performed over the interface with another layer because the stresses are discontinuous over this interface.

The moments were also calculated for the pg93 sample (PEI/glass) using the MPE approach, neglecting either $\varepsilon_{x}$ or $\kappa_{y}$ or the variation in material properties. The next section shows the corresponding influence on the calculated stresses.

\section{Stresses}

The stresses were calculated from the moment profiles using eqn (1) and a linear fit through the moment points as indicated in the moment profile figures. These calculations were performed according to the CLT and the MPE. The MPE calculations give the same results as the residual reference stress calculations in the original symmetric samples. Table 2 shows the

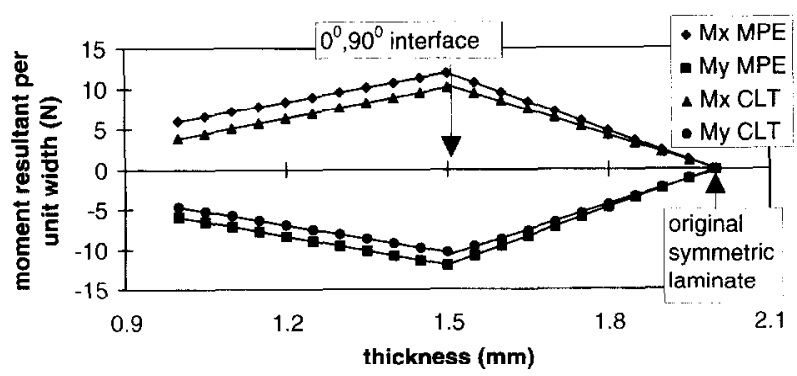

Fig. 6. Moment development after layer removal for the pg93 sample (PEI/glass).

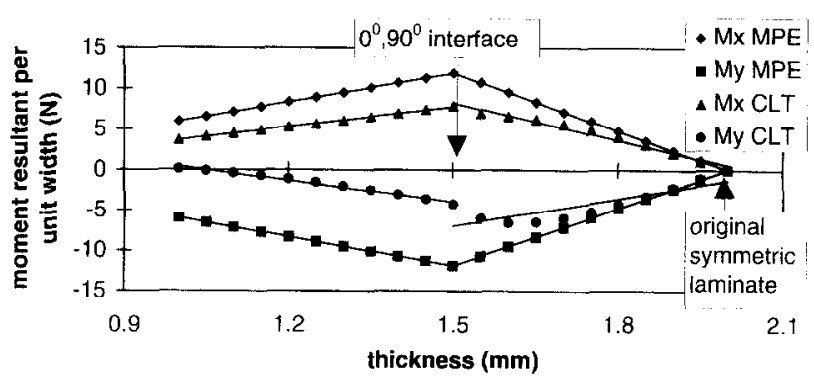

Fig. 7. Moment development after layer removal for the pg1010 sample (PEI/glass).

results for the four different crossply laminates. Table 2 also shows the results for the pg93 crossply laminate when any input parameter, as described before, is neglected.

\section{DISCUSSION}

The modified layer removal analysis using the MPE approach leads to the reference stresses for the four laminates. In all four laminates equilibrium in the stress profile is present. The errors in the CLT calculations arise because the moments are not calculated correctly. Therefore, the slope of the moment lines, as shown in Figs 6-9, will not be correct either. They both are present in eqn (1) from which the stresses are calculated. Further, the moment develop-

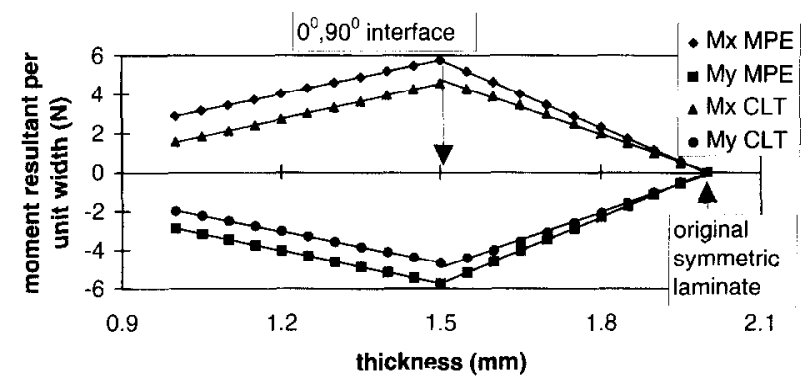

Fig. 8. Moment development after layer removal for the cg93-55 sample (PC/glass).

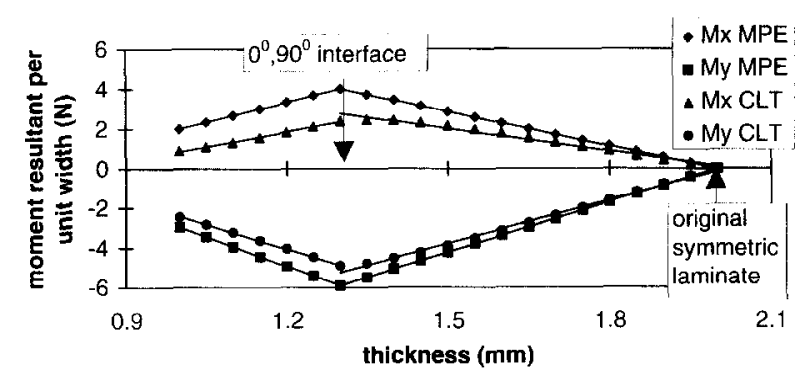

Fig. 9. Moment development after layer removal for the cg93-73 sample (PC/glass). 
Table 2. Stresses and errors from layer removal analysis validation for different examples

\begin{tabular}{|c|c|c|c|c|c|c|c|c|}
\hline Error in () & $\sigma_{x}$ & ayer) & & layer) & & layer) & $\sigma_{y}$ & layer) \\
\hline pg93 (MPE) & -23.8 & $(0)$ & 23.8 & $(0)$ & 23.8 & $(0)$ & -23.8 & $(0)$ \\
\hline pg93 (CLT) & -21.4 & $(-10.1)$ & 23.2 & $(-2.5)$ & 21.1 & $(-11.3)$ & -21.6 & $(-9.2)$ \\
\hline pg1010 (MPE) & -23.8 & (0) & 23.8 & $(0)$ & 23.8 & $(0)$ & -23.8 & $(\mathrm{O})$ \\
\hline pg 1010 (CLT) & -14.5 & $(-39.1)$ & 16.4 & $(-31.1)$ & 11.6 & $(-51.3)$ & -12.7 & $(-46.6)$ \\
\hline cg93-55 (MPE) & -11.5 & (0) & 11.5 & (0) & 11.5 & $(0)$ & -11.5 & $(0)$ \\
\hline cg93-55 (CLT) & -9.8 & $(14.8)$ & 11.0 & $(4.3)$ & 10 & $(13.0)$ & -10.3 & (10.4) \\
\hline cg93-73 (MPE) & -5.7 & (0) & 13.3 & $(0)$ & 8.4 & $(0)$ & -19.7 & $(0)$ \\
\hline cg93-73 (CLT) & -4.5 & $(-21.1)$ & 9.2 & $(-30.8)$ & 6.9 & $(-17.6)$ & -16.2 & $(-17.8)$ \\
\hline $\operatorname{pg} 93, \in_{x}{ }^{\prime \prime}=0$ & -40.7 & $(+72.7)$ & 41.1 & $(+71.0)$ & & & & \\
\hline $\operatorname{pg} 93, \kappa_{y}=0$ & -22.7 & $(-4.6)$ & 26.1 & $(+9.7)$ & & & & \\
\hline pg93, constant properties & 43.3 & $(+81.9)$ & 43.7 & $(+83.6)$ & & & & \\
\hline
\end{tabular}

ment should be linear resulting in constant stresses. However, Fig. 7 clearly shows a nonlinear moment development. Performing a higher-order polynomial fit in this case gives a better result, but leads to significant stress variation where the stresses should be constant.

Table 2 reveals several important points. The pg93 sample does not show significant errors for the CIT calculation compared to the MPE calculation. However, pg1010, where the sample dimensions are larger, viz. $100 \times 100 \mathrm{~mm}$, does show larger errors $(30-50 \%)$.

This effect of the sample dimensions can be illustrated with a diagram of the thermal curvatures in a composite plate after manulacture, as a function of the side length of the plate, calculated using the MPE. Such diagrams can be found in the literature [7-10] (Fig. 10).

The result of the CLT calculation is given by the curvatures at side length zero. The bifurcation point indicates the side length where the anticlastic shape of the laminate changes to a cylindrical shape. In this situation only one curvature is present and the other is suppressed.

Figure 10 clearly shows that for very small side lengths the CLT and MPE give the same results for the curvatures in the $x$ - and $y$-directions. For large side lengths, the CLT and MPE

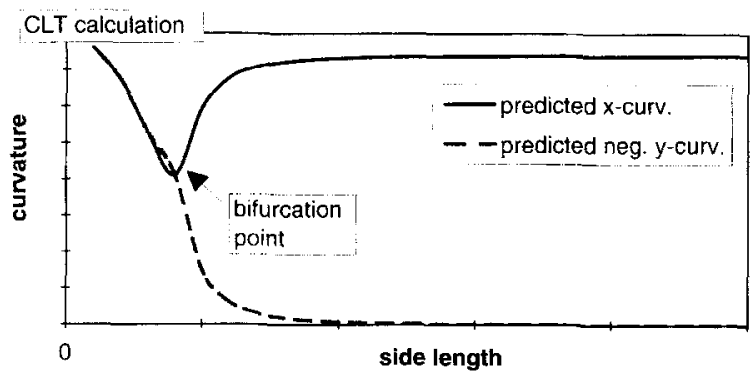

Fig. 10. A schematic diagram showing thermal curvatures calculated according to the MPE appoach as a function of the plate side length. give the same results only for curvature in the $x$ direction. For intermediate side lengths the errors between CLT and MPE are large. This implies that the sample dimension choice should not be arbitrary. For ease of analysis it is advisable to choose sample dimensions such that the linear CLT calculation approximates the MPE calculation. However, this might not always be possible owing to practical limitations.

The layer thicknesses in the laminate are also important. The cg93-55 sample shows the same errors in the CLT calculation as the pg93 sample. However the cg93-73 sample, where the layers are not of equal thicknesses, shows a much larger error in the CLT calculation.

The results of the $\mathrm{cg} 93-73$ sample (PC/glass) indicate large deformations in layer removal experiments. This cannot be neglected in the analysis. This laminate has material properties and a lay-up configuration which are very similar to injection-moulded plates. So, in principle, such analyses can be performed on injectionmoulded short-fibre-reinforced products.

Table 2 further shows that large errors $(70 \%)$ are introduced in the calculation of the stresses in the $x$-direction by neglecting the midplane strain $\varepsilon_{x}^{0}$. Therefore, this strain has to be measured in the layer removal experiments on samples with properties varying through the thickness. Similar to the $x$-direction, the strain $\varepsilon_{y}^{0}$ has to be measured for a correct calculation of the stresses in the $y$-direction (not illustrated here).

Table 2 shows that the influence of the curvature $k_{y}$ on the stresses in the $x$-direction is not large, but this strongly depends on the sample dimensions. Errors of $20-30 \%$ can be introduced for other sample dimensions. Using a very narrow specimen does not necessarily mean that the curvature in the $y$-direction can be neglected. It has been noticed by the authors 
that in some cases narrowing the $y$-dimension of the sample can lead to an increased influence of $\kappa_{y}$ on the stress calculation in the $x$-direction. Therefore, $\kappa_{y}$ must also be measured.

Table 2 shows the influence of approximating the varying material properties by constant material properties. The errors introduced are over $80 \%$ for the continuous-fibre-reinforced $\mathrm{PFI} / \mathrm{glass}$ sample. In addition, the typical injection-moulded sample PC/glass shows errors over $80 \%$ (not illustrated in this paper) when the material properties are assumed constant ( $E$-modulus 9.5 GPa and Poisson's ratio 0.3).

Finally, Table 2 shows that the presence of stress equilibrium in the layer removal results does not necessarily indicate that the magnitude of the stresses is correct. For example, the CLT calculation on the pg1010 sample leads to large errors in the stresses but a near stress equilibrium is present.

\section{CONCLUSIONS}

The layer removal method is further developed for use on composite materials with varying material properties through the thickness. The need to use the variation in material properties for injection-moulded and compression-moulded plates is clearly demonstrated. Using constant material properties in the analysis of the layer removal results leads to errors of over $80 \%$ in calculated stresses.

The deformations in layer removal on composite materials can become large and the CLT can no longer be used for a correct evaluation. Instead, the MPE approach described in this paper can be used for a accurate calculation of the stresses from layer removal experiments. In these calculations curvatures, as well as midplane strains, are needed and have to be measured. Neglecting strains or curvatures leads, in general, to unacceptable errors.

The CLT only provides a good approximation of the moments (induced by layer removal) leading to small errors in stress values in specific cases.

\section{REFERENCES}

1. Kim, K.-S., Hahn, H.T. and Croman, R.B., The effect of cooling rate on residual stress in a thermoplastic composite. J. Composite Technol. Res. 1989, 11, (2), 47-52.

2. Jeronimidis, G. and Parkyn, A.T., Residual stresses in carbon fibre-thermoplastic matrix laminates. J. Composite Mater. 1988, 22, 401-415.

3. Treuting, R.G. and Read, W.T., Jr., A mechanical determination of biaxial residual stress in sheet materials. J. Appl. Phys. 1951, 22, (2), 130-134.

4. White, J.R., On the layer removal analysis of residual stress, Part 1: Polymer mouldings with depth-varying Young's modulus. J. Mater. Sci. 1985, 20, 2377-2387.

5. Paterson, M.W.A. and White, J.R., Layer removal analysis of residual stress, Part 2: A new procedure for polymer mouldings with depth-varying Young's modulus. J. Mater. Sci. 1989, 24, 3521-3528.

6. Hyer, M.W., Calculations of the room-temperature shapes of unsymmetric laminates. $J$. Composite Mater. 1981, 15, 296-310.

7. Hyer, M.W., The room-temperature shapes of fourlayer unsymmetric cross-ply laminates. J. Composite Mater. 1982, 16, 318-340.

8. Jun, W.J. and Hong, C.S., Effect of residual shear strain on the cured shape of unsymmetric cross-ply thin laminates. Composite Sci. Technol. 1990, 38, 55-67.

9. Jun, W.J. and Hong, C.S., Cured shape of unsymmetric laminates with arbitrary lay-up angles. $J$. Reinf. Plast. Composites 1992, 11, 1352-1366.

10. Peeters, L.J.B., Powell, P.C. and Warnet, L.L., Thermally-induced shapes of unsymmetric laminates. $J$. Composite Mater: 1996, 30, (5), 603-626.

11. Powell, P.C., Engineering with Fibre-Polymer Laminates. Chapman and Hall, London, 1994.

12. Fenner, R.T., Engineering Elasticity. Ellis Horwood, Chichester, 1986.

13. Bijsterbosch, H. and Gaymans, R.J., Polyamide 6long glass fiber injection moldings. Polym. Composites $1995,16,(5), 363-369$.

14. Kenig, S., Fiber orientation development in molding of polymer composites. Polym. Composites 1986, 7, (1), 50-55.

15. Darlington, M.W. and Smith, A.C., Some features of the injection molding of short fiber reinforced thermoplastics in center sprue-gated cavities. Polym. Composites 1987, 8, (1), 16-21.

16. Bay, R.S., Tucker, C.L. and Davis, R.B., Effect of processing on fiber orientation in simple injection moldings. ANTEC 89 Techn. Pap. 1989, 35, 539542. 